\title{
Treatment with biologics during pregnancy in patients with rheumatic diseases
}

\section{Frauke Förger}

Department of Rheumatology, Immunology and Allergology, Inselspital, University Hospital, and University of Bern, Switzerland

Chronic inflammatory rheumatic diseases frequently affect women of the reproductive age. For these women family planning is an important issue in their life, yet this issue is characterized by a lot of concerns and conflicting information for both patients and doctors. To control disease activity and prevent joint or organ damage, a longterm immunosuppressive therapy is necessary in these patients. However, treatment during pregnancy concerns both, mother and unborn child. A pregnant woman with a chronic inflammatory rheumatic disease will always chose treatment with a pregnancy-compatible drug that shows a convincing safety profile for her and her unborn child. Disease activity and medication (like non-steroidal antirheumatic drugs or prednisolone) can have a negative influence on fertility and pregnancy outcome [1]. On the other hand, pregnancy can influence disease activity in a positive way (e.g. rheumatoid arthritis - RA) or in a negative way (e.g. axial spondyloarthritis - axSpA) [2].

For the management of patients with rheumatic diseases who plan to become pregnant, both, disease activity and therapeutic regimen have to be taken into consideration. In general, pregnancy can be planned in case of a stable inactive disease and in case of pregnancy-compatible drugs. The only biologic drugs that are regarded compatible with pregnancy are TNF inhibitors (TNFi) [3]. Based on the time of TNFi use during pregnancy, different points need to be considered. For the use of TNFi in the first trimester of pregnancy, the question arises whether TNFi increase the risk of miscarriage or malformation. In their systematic literature review the European League against Rheumatism (EULAR) analyzed the outcome of 2492 pregnancies exposed to TNFi [3]. No increased rate of miscarriages or congenital malformations could be found among those TNFi exposed pregnancies [3]. Accordingly, a recent systematic review and meta-analysis could not find an increased risk of miscarriages or malformation comparing TNFi users versus non-users [4]. For the use of TNFi in the second or third trimester of pregnancy, the potential of transplacental passage needs to be looked at. During the second and third trimester, IgG1 molecules are actively transported from the maternal circulation to the fetal circulation via binding to the neonatal Fc receptor on trophoblast cells [5]. The transport is most intense during the third trimester resulting in neonatal IgG1 levels that often exceed those of the mother. The different molecular structure of TNFi (etanercept, infliximab, adalimumab, golimumab and certolizumab) accounts for the differences in their transplacental transfer. Etanercept is a soluble fusion protein. Infliximab, adalimumab and golimumab are monoclonal IgG1 antibodies specific for TNF- $\alpha$. Certolizumab pegol is a pegylated Fc-free anti-TNF- $\alpha$ Fab'fragment which lacks the Fc moiety and thereby prevents its binding to placental FCRn. The binding affinity to the neonatal Fc receptor on placental trophoblast cells is highest for complete monoclonal antibodies such as infliximab and adalimumab, low for etanercept and absent for certolizumab [6]. Accordingly, the continuation of infliximab or adalimumab in the third trimester of pregnancy result in therapeutic levels in the newborn's cord blood that exceed the drug levels in the peripheral blood of their mothers [7, 8]. Maternal IgG1 has a prolonged half-life in the newborn, accordingly infliximab administered throughout the third trimester of pregnancy can still be detected in the serum of infants for up to 6-12 months following in-utero exposure $[9,10]$. The data for certolizumab and etanercept given to a pregnant patient during the third trimester show that only very low levels are found in the cord blood of the neonate for etanercept and below detection to minimal levels are found for certolizumab [5]. More data on the pharmacokinetic of certolizumab when given during pregnancy has been investigated recently in a prospective multicenter postmarketing study and will soon be available.

\section{Address for correspondence:}

Frauke Förger, Department of Rheumatology, Immunology and Allergology, Inselspital, University Hospital, and University of Bern, Switzerland, e-mail: Frauke.Foerger@insel.ch

Submitted: 5.04.2017; Accepted: 10.04.2017 
Follow-up data of the children's health after intra-uterine exposure to TNFi are still limited. Possible risks for the exposed children are infection and response to vaccination, especially live vaccines. Most data, including systematic reviews, could not show an increased risk of infection in the first year of life in the offspring of mothers who received TNFi during pregnancy [5]. However, in case of a combination therapy of the complete monoclonal TNFi adalimumab and infliximab and thiopurine in pregnant women with inflammatory bowel disease the relative risk for infection in exposed infants can be elevated as compared to TNFi monotherapy [10]. In can be expected that this aspect would be different for certolizumab since the transplacental transfer is completely different compared to adalimumab and infliximab, yet data on the long-term follow-up of certolizumab exposed infants are lacking.

With regard to vaccination in children exposed to TNFi before gestational week 22, the normal vaccination program can be followed [3]. In children exposed to TNFi during the late second or third trimester it is recommended to wait 6 months following birth before administering any live vaccines (like BCG) [3].

Together, TNFi are the best studied biologics in pregnancy. They do not increase the risk for miscarriages or congenital malformations and therefore, appear reasonably safe if used during the first half of pregnancy. Due to low rate of transplacental passage, etanercept and certolizumab may be considered for use throughout pregnancy if indicated [3].

The author declares no conflict of interest.

\section{References}

1. Brouwer J, Hazes JM, Laven JS, Dolhain RJ. Fertility in women with rheumatoid arthritis: influence of disease activity and medication. Ann Rheum Dis 2015; 74: 1836-1841.

2. Ostensen M, Villiger PM, Forger F. Interaction of pregnancy and autoimmune rheumatic disease. Autoimmun Rev 2012; 11: A437-446.

3. Gotestam Skorpen C, Hoeltzenbein M, Tincani A, et al. The EULAR points to consider for use of antirheumatic drugs before pregnancy, and during pregnancy and lactation. Ann Rheum Dis 2016; 75: 795-810.

4. Komaki F, Komaki Y, Micic D, et al. Outcome of pregnancy and neonatal complications with anti-tumor necrosis factor-alpha use in females with immune mediated diseases; a systematic review and meta-analysis. J Autoimmun 2017; 76: 38-52.

5. Forger F, Villiger PM. Treatment of rheumatoid arthritis during pregnancy: present and future. Exp Rev Clin Immunol 2016; 12: 937-944.

6. Porter C, Armstrong-Fisher S, Kopotsha T, et al. Certolizumab pegol does not bind the neonatal Fc receptor (FCRn): Consequences for FcRn-mediated in vitro transcytosis and ex vivo human placental transfer. J Reprod Immunol 2016; 116: 7-12.

7. Zelinkova Z, de Haar C, de Ridder L, et al. High intra-uterine exposure to infliximab following maternal anti-TNF treatment during pregnancy. Aliment Pharmacol Ther 2011; 33: 1053-1058.

8. Zelinkova Z, van der Ent C, Bruin KF, et al. Effects of discontinuing anti-tumor necrosis factor therapy during pregnancy on the course of inflammatory bowel disease and neonatal exposure. Clin Gastroenterol Hepatol 2013; 11: 318-321.

9. Vasiliauskas EA, Church JA, Silverman N, et al. Case report: evidence for transplacental transfer of maternally administered infliximab to the newborn. Clin Gastroenterol Hepatol 2006; 4: 1255-1258.

10. Julsgaard M, Christensen LA, Gibson PR, et al. Concentrations of Adalimumab and Infliximab in Mothers and Newborns, and Effects on Infection. Gastroenterology 2016; 151: 110-119. 